\title{
LD $_{2}$ SNPing: linkage disequilibrium plotter and RFLP enzyme mining for tag SNPs
}

\author{
Hsueh-Wei Chang1,2,3, Li-Yeh Chuang*4, Yan-Jhu Chang6, Yu-Huei Cheng, \\ Yu-Chen Hung ${ }^{1}$, Hsiang-Chi Chen ${ }^{5}$ and Cheng-Hong Yang*6
}

\begin{abstract}
Address: ${ }^{1}$ Department of Biomedical Science and Environmental Biology, Kaohsiung Medical University, Kaohsiung, Taiwan, ${ }^{2}$ Graduate Institute of Natural Products, College of Pharmacy, Kaohsiung Medical University, Kaohsiung, Taiwan, ${ }^{3}$ Center of Excellence for Environmental Medicine, Kaohsiung Medical University, Kaohsiung, Taiwan, ${ }^{4}$ Department of Chemical Engineering, I-Shou University, Kaohsiung, Taiwan, ${ }^{5}$ Institute of Molecular and Cellular Biology, National Tsing Hua University, Hsinchu, Taiwan and ${ }^{6}$ Department of Electronic Engineering, National Kaohsiung University of Applied Sciences, Kaohsiung, Taiwan

Email: Hsueh-Wei Chang - changhw@kmu.edu.tw; Li-Yeh Chuang* - chuang@isu.edu.tw; Yan-Jhu Chang - ck713112@hotmail.com; YuHuei Cheng - yuhuei.cheng@gmail.com; Yu-Chen Hung - ok7836791@hotmail.com; Hsiang-Chi Chen - chess0830@gmail.com; ChengHong Yang* - chyang@cc.kuas.edu.tw

* Corresponding authors
\end{abstract}

Published: 6 June 2009

BMC Genetics 2009, 10:26 doi:10.1186/147|-2156-10-26

This article is available from: http://www.biomedcentral.com/I47/-2/56/I0/26

(C) 2009 Chang et al; licensee BioMed Central Ltd.

This is an Open Access article distributed under the terms of the Creative Commons Attribution License (http://creativecommons.org/licenses/by/2.0), which permits unrestricted use, distribution, and reproduction in any medium, provided the original work is properly cited.
Received: II November 2008

Accepted: 6 June 2009

\begin{abstract}
Background: Linkage disequilibrium (LD) mapping is commonly used to evaluate markers for genome-wide association studies. Most types of LD software focus strictly on LD analysis and visualization, but lack supporting services for genotyping.

Results: We developed a freeware called LD $_{2}$ SNPing, which provides a complete package of mining tools for genotyping and LD analysis environments. The software provides SNP ID- and gene-centric online retrievals for SNP information and tag SNP selection from dbSNP/NCBI and HapMap, respectively. Restriction fragment length polymorphism (RFLP) enzyme information for SNP genotype is available to all SNP IDs and tag SNPs. Single and multiple SNP inputs are possible in order to perform LD analysis by online retrieval from HapMap and NCBI. An LD statistics section provides $D, D^{\prime}, r^{2}, \delta_{Q}, \rho$, and the $P$ values of the Hardy-Weinberg Equilibrium for each SNP marker, and Chi-square and likelihood-ratio tests for the pair-wise association of two SNPs in LD calculation. Finally, 2D and 3D plots, as well as plain-text output of the results, can be selected.

Conclusion: $\mathrm{LD}_{2}$ SNPing thus provides a novel visualization environment for multiple SNP input, which facilitates SNP association studies. The software, user manual, and tutorial are freely available at http://bio.kuas.edu.tw/LD2NPing.
\end{abstract}

\section{Background}

Single nucleotide polymorphisms (SNPs) are very important markers for disease [1] and cancer [2] association studies. The number of identified SNPs is currently estimated to be about 3.1 million [3]. Identification of asso- ciations by statistical analyses of SNP data is challenging due to the large number of SNPs involved.

Linkage disequilibrium (LD) is one of the most commonly used methods when choosing informative SNPs 
that represent the original SNP distribution in a genome for genome-wide association studies. LD mappings are commonly used to evaluate markers across large data sets. Given the vast amount of data in association studies, visualization of the LD results in graphical form rather than text form facilitates the interpretation of the results considerably [4].

Many types of visualization software for LD have been developed, e.g. LDA [5], Haploview [6], and JLIN [7]. Although these tools have made valuable contributions to LD visualization and analysis, they lack many services and tools for users to generate genotype data for LD analysis. Without the actual data set itself, users are unable to perform LD analysis. However, many types of software exist which provide information for genotyping, e.g. the SNPlex genotyping system [8], SNP cutter [9], SNP-RFLPing [10], and V-MitoSNP [11]. These programs do not include an LD function though. It is thus still difficult for researchers to narrow down the number of SNPs for performing SNP genotyping. A common way of identifying tag SNPs of the genes of interest is to check the HapMap website http://www.hapmap.org[12]. Currently available tools, however, are not well integrated, but rather are independent programs.

We have thus integrated an SNP genotyping service and $\mathrm{LD}$ visualization/analysis tool in a single program to provide a single platform for tag SNP selection, SNP genotyping, and LD analysis. This platform, $\mathrm{LD}_{2}$ SNPing, furthermore provides a novel function for multiple SNP inputs in order to directly plot the LD. The user can input SNPs of interest and calculate the LD measurement for SNP selection before the genotyping process. This standalone JAVA-based visualisation tool greatly facilitates preparation of the genotype data and increases the performance of LD analyses.

\section{Implementation}

$\mathrm{LD}_{2}$ SNPing is a Java-based software, which is implemented under the Java Runtime Environment (JRE) and Java $3 \mathrm{D}$. The $\mathrm{LD}$ statistics program calculates $D, D^{\prime}, r^{2}, \delta_{\mathrm{Q}^{\prime}}$ and $\rho$ values, as well as the $P$ value of Hardy-Weinberg Equilibrium (HWE-P) calculations for each SNP marker. $\mathrm{LD}_{2}$ SNPing provides the $P$ value of the Chi-square test and $P$ value of the likelihood-ratio test for the pair-wise association of two SNPs are also provided in the LD calculation. $\mathrm{LD}_{2} \mathrm{SNPing}$ processes genotype data and estimates pair-wise loci haplotype frequencies of the sample using an expectation-maximization algorithm (EM) [13]. Except the exact tests of HWE [14] is implemented in $\mathrm{LD}_{2} \mathrm{SNPing}$, the equations used in these calculations are listed in the appendix of the user manual as described by LDA [5].
In visualization of $\mathrm{LD}$ plot, the $\mathrm{LD}_{2} \mathrm{SNPing}$ software provides SNPs with a minor allele frequency (MAF) value greater than 0.01. All the MAF and HWE-P values for these SNPs are provided in the text window.

The SNP genotype information and the tag SNPs are retrieved online from dbSNP version BUILD 129 of NCBI [15]http://www.ncbi.nlm.nih.gov/SNP/[16] and HapMap http://www.hapmap.org version HapMap Data Rel 23a/phaseII Mar08, on the NCBI B36 assembly, dbSNP b126 [12], respectively. Online retrieval for SNP genotype information from NCBI using SNP ID and gene input is similar to the function described in the SNP-Flankplus [17] and SNP ID-info [18]. The default setting for the minor allele frequency (MAF) cut-off in tag SNP from HapMap is 0.2. Four populations, CEU, CHB, JPT, and YRI (Caucasian, Han-Chinese, Japanese and Sub-Saharan African, respectively) are selectable during tag SNP retrieval from HapMap. The retrieved data are the most up-to-date data available. The RFLP database structure is based on REBASE http://www.rebase.org[19] version 610 . The RFLP mining function for the selected SNP is provided by the SNP-RFLPing [10], which is integrated in the $\mathrm{LD}_{2}$ SNPing.

A demonstration and user manual of the $\mathrm{LD}_{2}$ SNPing software are available as a free download from http:// bio.kuas.edu.tw/LD2SNPing. Many animations explaining how to use the $\mathrm{LD}_{2}$ SNPing software are provided on the homepage and embedded in the user manual (see Additional file 1) as tutorials.

\section{Results}

Data import formats: File input

$\mathrm{LD}_{2}$ SNPing accepts four different input file formats, namely two Excel (.xls and .cvs), Word (.doc) and NotePad (.txt) formats. The first and second rows for each file are reserved for the user-defined SNP name and the distance between SNPs (optional), respectively. Individual genotypes accept the following formats: NN, N_N, and N/ $\mathrm{N}$ ( $\mathrm{N}$ is one of four possible nucleotides). If the input file is missing a genotype, it is automatically bypassed in $\mathrm{LD}_{2}$ SNPing processing without interference. Some example files for testing are available in the example file folder of the $\mathrm{LD}_{2}$ SNPing software package.

\section{Data import formats: rsID input}

$\mathrm{LD}_{2}$ SNPing provides the rsID\# input for online retrieval of individual SNP information from the dbSNP of the NCBI (Figure 1A).

\section{Data import formats: Gene input}

$\mathrm{LD}_{2} \mathrm{SNPing}$ accepts gene name (HUGO, Human Genome Organization) input to provide tag SNPs through online retrieval from HapMap (Figure 1B). 


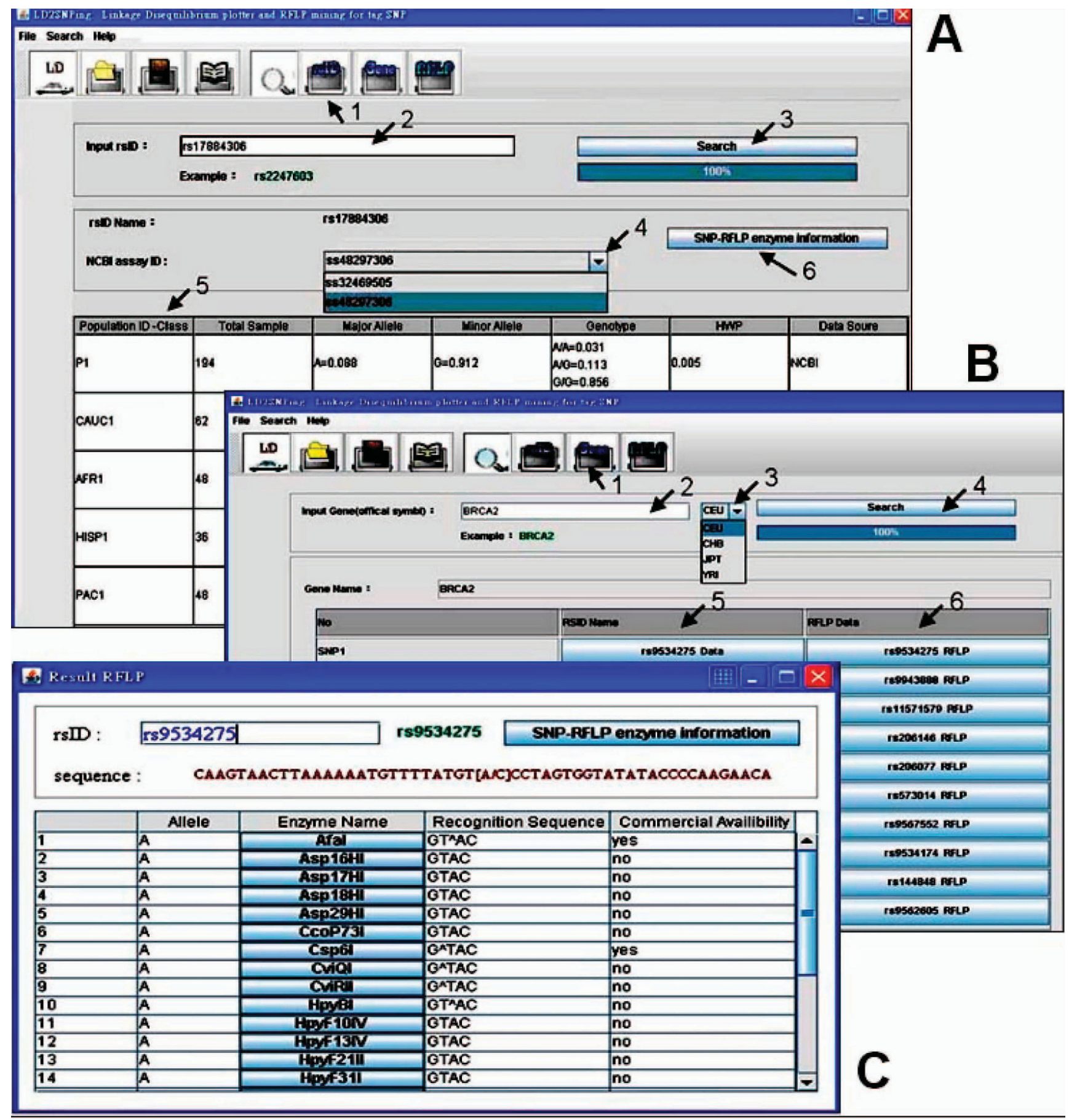

Figure I

Retrieval of SNP information by LD 2 SNPing. (A) rsID\# input to retrieve SNP genotype information from dbSNP in NCBI. Arrows I to 5 indicate the steps to retrieve SNP information. The different ssID\#s for the same rsID\# can be selected as indicated by the arrow at line 4 . Population class, total sample, major allele, minor allele, genotype frequencies, $P$ value of Hardy-Weinberg equilibrium (HWE-P) and data source are provided as indicated by arrow 5 . The RFLP information for inputting the SNP is provided (arrow line 6). (B) Gene input to retrieve tag SNPs from HapMap [12]. Four populations can be selected. The RFLP information for each tag SNP is also provided. (C) Restriction enzyme mining for RFLP in SNP genotype. Results similar to the RFLP information described in Figures IB and IC are shown. 


\section{LD-free function: Retrieval of individual SNP information from NCBI}

In Figure 1A, the SNP (rs17884306) information for all populations of the dbSNP is provided (P1, CAUC1, AFR1, HISP1, and PAC1). The ssID\#s (ss32469505 and ss48297306) for the corresponding rsID\# (rs17884306) can be selected by using the pull-down window.

\section{LD-free function: Gene input for finding rsID data of tag SNP}

In Figure $1 \mathrm{~B}, \mathrm{LD}_{2} \mathrm{SNPing}$ provides the tag SNP information through HapMap by gene input. The example shown is BRCA2. The tag SNP candidates provided by $\mathrm{LD}_{2}$ SNPing are completely matched with those of HapMap (shown in the user manual). HapMap-CEU, HCB, JPT and YRI are acceptable for selection.

\section{LD-free function: RFLP enzyme mining tool}

Before performing LD analysis, it is necessary to collect SNP genotype data for genes of interest, such as the SNP ID input (Figure 1A) and tag SNPs (Figure 1B).

$\mathrm{LD}_{2}$ SNPing executes RFLP restriction enzyme mining upon clicking of the RFLP box indicated by arrow 6 of Figure $1 \mathrm{~A}$ and arrow 5 of Figure $1 \mathrm{~B}$. RFLP results are shown in the format pictured in Figure $1 \mathrm{C}$, in which restriction enzyme information for SNPs of interest (here, rs9534275) are shown. Information about alleles, enzyme name, the recognition sequence and commercial availability is provided.

\section{LD function: Input formats for 2D analysis}

$\mathrm{LD}_{2}$ SNPing provides for file input and sample file input to perform LD analysis and visualization (numbers 1 and 2 of Figure 2A, respectively). Moreover, $\mathrm{LD}_{2} \mathrm{SNPing}$ provides for online retrieval of multiple SNP inputs for LD measurement, prediction and visualization (numbers \#1 to \#8 of Figures $2 \mathrm{~A}$ and $2 \mathrm{~B}$ ). For convenience, the LD for any SNPs located on the same chromosome can be directly analyzed. Figure 2B shows the single SNP rsID\# (rs2078486), which has six different ssID\#s from different data sources. For example, ss20037931 has HapMapCEU, HCB, JPT and YRI as data sources. Different data sources have different genotype frequencies for the same SNP rsID\# due to the different data sets. The data was retrieved online from dbSNP of NCBI and confirmed to match (shown in user manual). Both file input and multiple SNP input lead to results similar with those shown in Figure 3, although the color pattern is different (described later).

\section{LD function: 2D-LD graph}

The distance between SNPs supplied in the input file can be optionally displayed or hidden (number 1 of Figure $3 \mathrm{~A})$. This distance is shown next to the diagonal line as a numerical value. By clicking on the "select scope" (number 2 of Figure 3A) and "repaint" (number 8 of Figure $3 \mathrm{~A}$ ) buttons, a user can limit the number of SNPs shown to only those of interest. This view can be reversed by clicking on the "restore scope" (number 3 of Figure 3A) button. The parameters for LD measurement are selected by the two axes named "left and right LD measure" (numbers 4 and 5 of Figure 3A, respectively). Different color schemes for each of the statistics can be selected (numbers 6 and 7 of Figure 3A). Moreover, $\mathrm{LD}_{2}$ SNPing provides a window for the minor allele frequency (MAF) value and HWE- $P$ values for each analyzed SNP when LD analysis is performed (not shown). A more detailed description is given in the user manual.

\section{LD function: Data analysis of LD information}

$\mathrm{LD}_{2}$ SNPing provides spontaneous analysis of the LD measurements for each pair-wise SNPs by clicking. For example, a text window (Figure 4A) will open when the arrow located in the box of SNP5 vs. SNP2 (Figure 3A) is clicked. In Figure 4A, the allele/haplotype frequencies, Chi-square $P$ value, likelihood-ratio $P$ value and all LD statistics $\left(D, D^{\prime}, r^{2}, \delta_{Q^{\prime}}\right.$ and $\left.\rho\right)$ of paired SNPs are provided. These values are matched to the LDA software [5] (not shown).

In addition, $\mathrm{LD}_{2} \mathrm{SNPing}$ provides graphic analyses, such as grids and pie3D graphs, to supplement the 2D-LD visualization and analysis (numbers of 10 and 11 of Figure $3 \mathrm{~A}$ ). The results are shown in the user manual.

\section{LD function: 3D-LD graph}

The $3 \mathrm{D}$ visualization of $\mathrm{LD}$ is performed by clicking on the icon for number 13 in Figure 3A. It is the same as in the 2D-LD plot except for the color patterns and the color ranges. In $\mathrm{LD}-3 \mathrm{D}$, the distance and $\mathrm{LD}$ measurement values are indicated by the height in the diagonal line (Figure $3 \mathrm{~B})$. Users can toggle between the $2 \mathrm{D}-\mathrm{LD}$ view or close the analysis by clicking on the icon for numbers 12 and 9 of Figure 3A, respectively.

\section{Data export}

All the analyzed results can be saved as tab-delimited text files (.txt) and graphic files (.jpg) for convenience. The LD parameters are exported to a single file. Figure $4 \mathrm{~B}$ shows a sample test result for "LD measure data", $D^{\prime}$. All the $D^{\prime}$ values for each SNP are listed pairwise, a common publishing format. Other LD parameters are not shown here, but are available in the user manual.

\section{Discussion \\ Comparison of some LD software}

Many kinds of software for LD visualisation are freely available. LDA [5], Haploview [6], and JLIN [7] were written in Java to implement LD analyses. A comparison of 


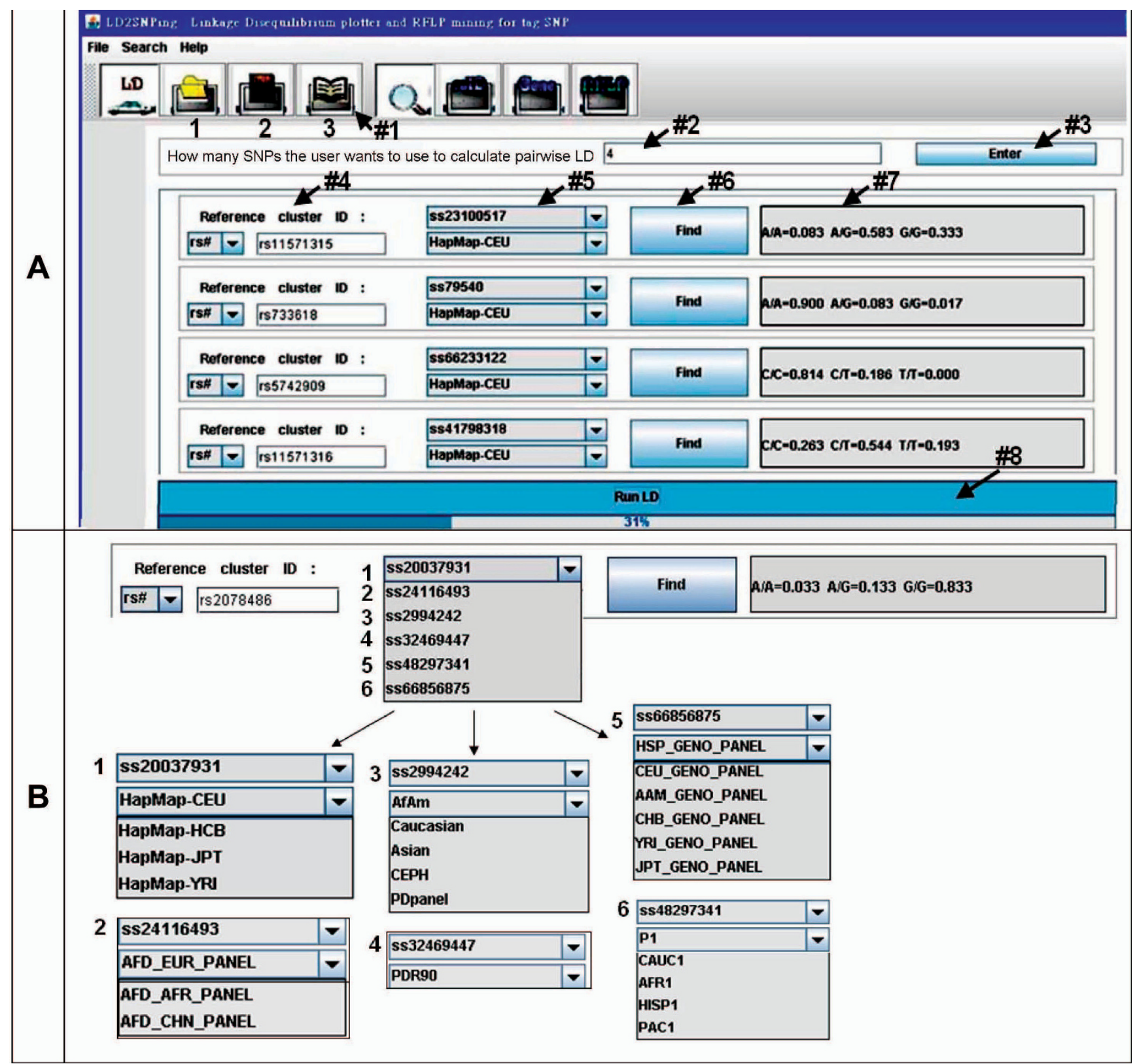

\section{Figure 2}

Online retrieval of multiple SNP input for LD measurement, prediction, and visualization. (A) Three LD functions are available in $\mathrm{LD}_{2}$ SNPing: I) user-defined files, 2) sample files, and 3) multiple SNPs. The input steps for multiple SNPs are demonstrated by arrows \#I to \#8. Four SNPs, rsII57I3I5, rs7336I8, rs5742909, and rsII57I316, are used as examples. All the ssID\#s with HapMap-CEU are selected. All selected SNP genotypes are provided after manually clicking the "Find" box. Similar results are shown in Figures 3 and 4. (B) Multiple choices for LD analysis of single rsID\# SNP. In the example, rs2078486, six ssID\#s are available. Each ssID\# possesses different SNP data sets as indicated by numbers I to 6 . The data is retrieved from NCBI and HapMap.

the different LD software is shown in Table 1. LDA and JLIN provide many LD measurements, but LDA offers only limited options for visualization of the results. Some LD parameters are not provided by Haploview, e.g. $\delta_{Q}$ and $\rho$ values.
Generally, SNP genotyping has to be performed to generate the SNP genotypes needed for LD analysis. Before performing LD analysis, however, all of the available $\mathrm{LD}$ software platforms only provide LD measurements without providing supporting functions, such as tag SNP min- 


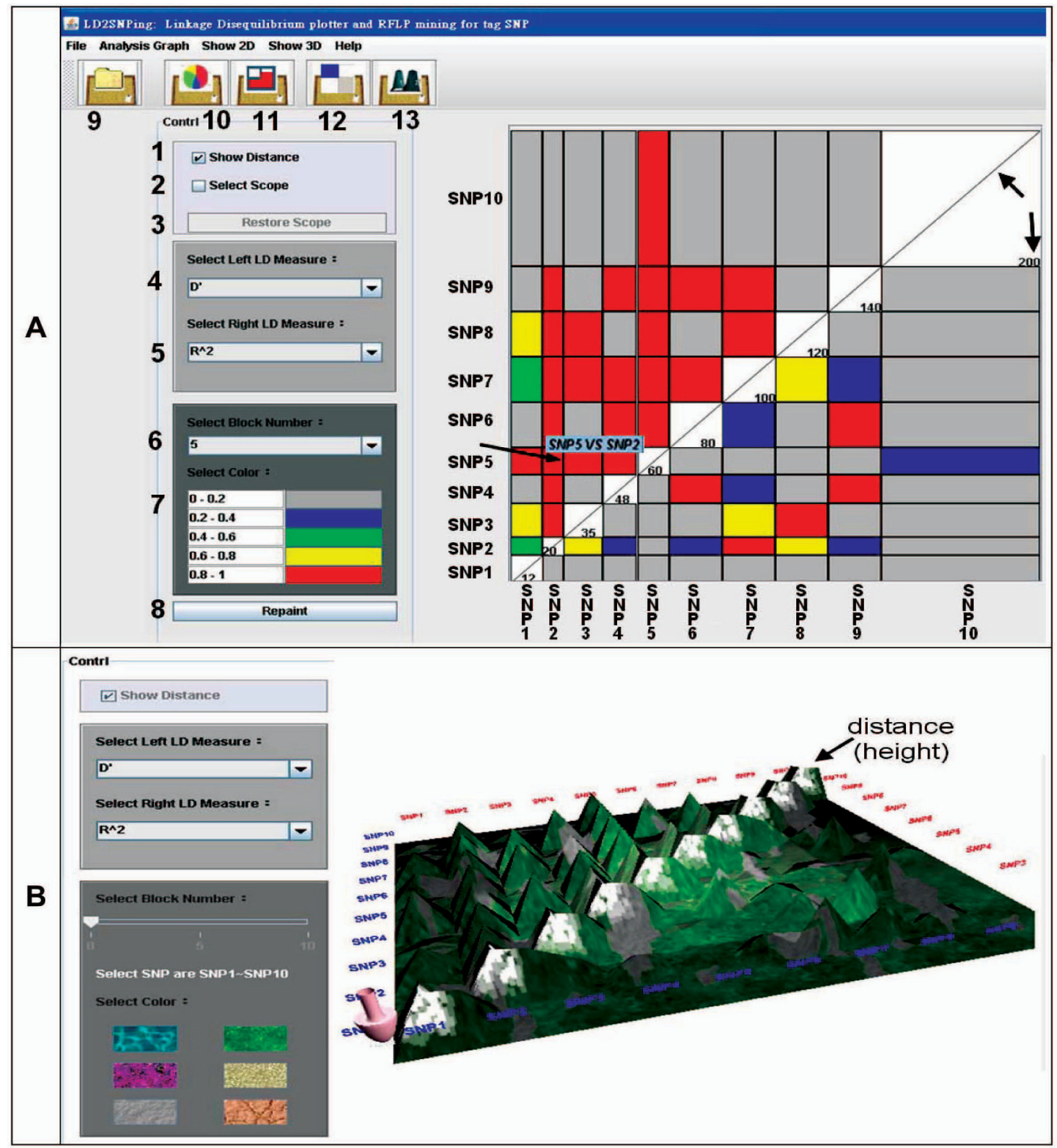

Figure 3

Available functions in the control panel of 2D-LD and 3D-LD plots. (A) The screen view after performing the LD calculation. The control panel contains eight functions I) Select display of distance. 2) Scope selection of the 2D-LD plot. 3) Restore scope selection. 4) Pull-down selection of the left (vertebrate axis) LD measure. 5) Pull-down selection of the right (horizontal axis) LD measure. 6) The different colours of the blocks in the diagram in Figure 3 indicate a different degree of interaction of the SNPs. 7) User-defined colour selection. 8) Repainting of 2D-LD profile. The arrows indicate the distance between SNPs. The functions marked 9 to 13 represent indicate closing a file, pie3D graph, bar graph, homing in on 2D-LD plot, and 3D-LD visualization, respectively. The arrow pointing to the box of SNP5 vs. SNP2 is displayed by mousing-over. (B) 3D-LD visualization. The distances between SNPs are indicated by the height of the diagonal line. 


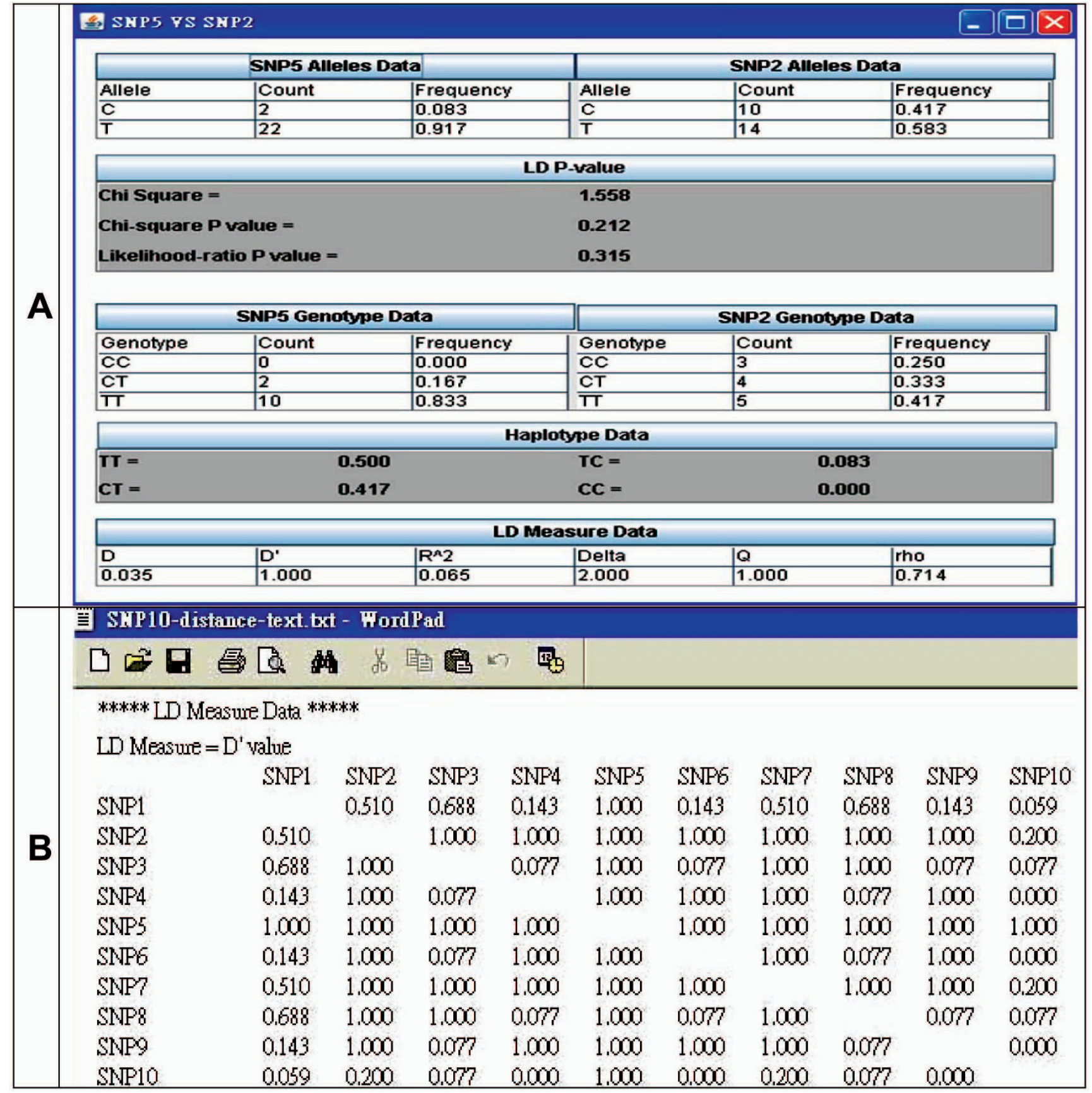

Figure 4

Output sample of LD measurement information. (A) Clicking on the box of the 2D-LD plot shows all LD-related information. SNPI0-distance.xls in example file folder of $\mathrm{LD}_{2}$ SNPing software is used as an example. Upon clicking the box of interest, such as the Box (SNP5 vs. SNP2) in Figure 3A (indicated by arrow line), the text data of this pair of SNPs for LD-related information is provided such as $D, D^{\prime}, r^{2}, \delta_{Q}, \rho$, Chi-square and likelihood-ratio $P$ values, and the SNP and haplotype frequencies. (B) Output for LD-related values. Here, the $D^{\prime}$ value is shown as a representative example; other values are shown in the user manual. This file belongs to a WordPad file which is saved for output LD measurement data. It can be opened by WordPad and Microsoft Word. 
Table I: Comparison of some LD software platforms

\begin{tabular}{|c|c|c|c|c|}
\hline & LDA & JLIN & Haploview & $\mathrm{LD}_{2}$ SNPing \\
\hline Input file formats & txt & csv & ped, info, txt & $x l s$, csv, txt, doc \\
\hline Output file formats & print & pdf, eps, png, txt & png, txt & jpg, txt \\
\hline$D, D^{\prime}, r^{2}, P$ & $\checkmark$ & $\lambda$ & 2 & 1 \\
\hline$\delta_{Q}, \rho$ & $\checkmark$ & $\checkmark$ & & $\checkmark$ \\
\hline Hardy-Weinberg equilibrium & $\checkmark$ & $\checkmark$ & $\checkmark$ & $\checkmark$ \\
\hline Genotype/haplotype frequency & & $\checkmark$ & $\checkmark$ & $\checkmark$ \\
\hline Tag SNP mining by gene input & & & & $\checkmark$ \\
\hline SNP information retrieval by rsID input & & & & $\checkmark$ \\
\hline Enzyme mining for RFLP genotyping & & & & $\checkmark$ \\
\hline Online retrieval of multiple SNPs for LD plot & & & & $\checkmark$ \\
\hline 2D distance & & $\checkmark$ & $\checkmark$ & $\checkmark$ \\
\hline 2D graph visualization & $\checkmark$ & $\checkmark$ & $\checkmark$ & $\checkmark$ \\
\hline 2D graph/text data output & & $\checkmark$ & $\checkmark$ & $\checkmark$ \\
\hline 3D distance/graph visualization & & & & $\checkmark$ \\
\hline
\end{tabular}

ing by gene input, retrieval of SNP information, or RFLP enzyme mining for genotype. These supporting functions are provided in $\mathrm{LD}_{2} \mathrm{SNPing}$ (Table 1 ). Moreover, $\mathrm{LD}_{2}$ SNPing allows for input of multiple SNPs for LD analysis (Figure 2). The genotype information of input SNPs are retrieved online from NCBI and HapMap. Therefore, users have an overview of the $\mathrm{LD}$ analysis for the input SNPs without performing prior SNP genotyping or inputting the genotype file. In contrast, Haploview provides many SNPs and users must manually select SNPs of interest. If the SNPs of interest are distributed widely over the chromosome, the SNP panel contains a large number of SNPs. Haploview thus only indirectly provides LD analysis for multiple SNPs.

\section{Tag SNP selection}

Tag SNP selection candidates from different operation times in HapMap may not be consistent due to changes made in the built-in greedy algorithm. Some tag SNPs may or may not be found again in subsequent tests. For example, tag SNP selection by inputting gene BRCA2 to HapMap under MAF $=0.2$ yields two tag SNP sets: 1 ) rs9534342, rs9943888, rs11571662, rs206120, rs206342, rs542551, rs9567552, rs206079, rs9562605, and rs14448 and 2) rs9534275, rs9943888, rs11571579, rs206146, rs206077, rs573014, rs9567552, rs9534174, rs144848, and rs9562605.

\section{Restriction enzyme mining for RFLP}

The $\mathrm{LD}_{2}$ SNPing provides the SNP ID searching to online retrieval to dbSNP in NCBI for RFLP analysis. However, the RFLP analysis for SNP ID input may be unable to provide the restriction enzyme information due to the nature of SNP itself. For example, the sequence information for rs9943888 and rs11571579 are retrieved successfully in $\mathrm{LD}_{2}$ SNPing but only rs 11571579 has the suitable restriction enzymes to mine (not shown). This is the nature for the SNP itself but not the RFLP analysis system error. For the wet experiment of PCR-RFLP, the users need the primer design software such as the "Prim-SNPing" [20] for primer design for SNP-RFLP and "SNP-Flankplus" [17] for the retrieval of SNP flanking sequence for primer design.

\section{Conclusion}

$\mathrm{LD}_{2}$ SNPing has the following characteristics: 1) it provides a search function for online retrieval of SNP information from dbSNP of NCBI; 2) it provides gene-centric tag SNP selection through online retrieval from HapMap; 3) all the SNP IDs and tag SNPs are processed to mine RFLP restriction enzymes for SNP genotype; 4) it provides LD measurements for $D, D^{\prime}, r^{2}, \delta_{Q^{\prime}}$ and $\rho$, along with the $P$ value of the Hardy-Weinberg Equilibrium for each SNP marker and the $P$ values of the Chi-square and likelihoodratio tests for the pair-wise association of two SNPs in LD calculation; 5) it accepts multiple SNP inputs to perform LD analysis by online retrieval from HapMap and NCBI; 6) it presents both $2 \mathrm{D}$ and $3 \mathrm{D}$ visualization with LDrelated measurements shown on the graphs; 7 ) it provides both graphic and plain-text outputs for LD analysis. In conclusion, $\mathrm{LD}_{2}$ SNPing is a novel and integrated visualisation software designed to provide the user with the tools necessary for genotyping and $\mathrm{LD}$ analysis. It provides a simple and user-friendly interface with integrated functions for retrieval of SNP information, LD statistical calculation, analysis and visualization.

\section{Availability and requirements}

Project name: LD2SNPing: Linkage disequilibrium plotter and RFLP enzyme mining for tag SNPs

Project home page: http://bio.kuas.edu.tw/LD2SNPing/ with software and user manual for download.

Operating system(s): Platform-independent

Programming language: Java 
Other requirements: Java 1.5.0 or higher

License: Free for non-commercial use

Any restrictions to use by non-academics: Please contact corresponding author.

\section{Abbreviations}

SNP: single nucleotide polymorphism; LD: linkage disequilibrium; RFLP: restriction fragment length polymorphism; HWE: Hardy-Weinberg Equilibrium; EM: expectation-maximisation algorithm; HUGO: Human Genome Organization; MAF: minor allele frequency.

\section{Authors' contributions}

HWC and LYC wrote the manuscript. LYC provides the genomics information and LD-related statistics. YJC designed and developed the Java implementation of the underlying algorithms and GUI. YHC improved the RFLP performance and online retrieval for SNP information. HWC instructed HCH and HCC regarding software testing, improvement, and animation preparation. CHY coordinated and oversaw this study. All authors read and approved the final manuscript.

\section{Additional material}

\section{Additional File 1}

User manual for $L D_{2}$ SNPing. User manual for $L D_{2} S N P i n g$.

Click here for file

[http://www.biomedcentral.com/content/supplementary/1471-

2156-10-26-S1.pdf]

\section{Acknowledgements}

This work was partly supported by the National Science Council in Taiwan under grants 97-23 II-B-037-003-MY3, 96-222I-E-2I4-050-MY3, NSC9623II-B037-002, 96-2622-E-I5I-019-CC3, NSC96-2622-E2 I4-004-CC3, KMU-EM-97-I.Ib, and KMU-EM-98-I.4.

\section{References}

I. Shastry BS: SNPs in disease gene mapping, medicinal drug development and evolution. Journal of human genetics 2007, 52(II):87I-880.

2. Zheng SL, Sun J, Wiklund F, Smith S, Stattin P, Li G, Adami HO, Hsu $\mathrm{FC}$, Zhu $Y$, Balter K, et al:: Cumulative association of five genetic variants with prostate cancer. N Engl J Med 2008, 358(9):910-919.

3. Frazer KA, Ballinger DG, Cox DR, Hinds DA, Stuve LL, Gibbs RA, Belmont JW, Boudreau A, Hardenbol P, Leal SM, et al.: A second generation human haplotype map of over 3.I million SNPs. Nature 2007, 449(7 | 64):85|-86I.

4. Carter K, Bellgard M: MASV-Multiple (BLAST) Annotation System Viewer. Bioinformatics (Oxford, England) 2003, 19(I723 I3-23 I5 [http://cbbc.murdoch.edu.au/projects/masv/].

5. Ding K, Zhou K, He F, Shen Y: LDA-a java-based linkage disequilibrium analyzer. Bioinformatics (Oxford, England) 2003, 19(162 |47-2/48 [http://www.chgb.org.cn//da/lda.htm]

6. Barrett JC, Fry B, Maller J, Daly MJ: Haploview: analysis and visualization of LD and haplotype maps. Bioinformatics (Oxford, Eng- land) 2005, 2 I (2263-265 [http://www.broad.mit.edu/mpg/haploview/ ].

7. Carter KW, McCaskie PA, Palmer LJ: JLIN: a java based linkage disequilibrium plotter. BMC bioinformatics 2006, 7:60 [http:// www.genepi.org.au/projects/jin].

8. Tobler AR, Short S, Andersen MR, Paner TM, Briggs JC, Lambert SM, Wu PP, Wang Y, Spoonde AY, Koehler RT, et al.: The SNPlex genotyping system: a flexible and scalable platform for SNP genotyping. J Biomol Tech 2005, 16(4):398-406.

9. Ding K, Zhang J, Zhou K, Shen Y, Zhang X: htSNPer I.0: software for haplotype block partition and htSNPs selection. BMC bioinformatics 2005, 6:38 [http://www.chgb.org.cn/htSNPer/htSN Per.html].

10. Chang HW, Yang CH, Chang PL, Cheng YH, Chuang LY: SNPRFLPing: restriction enzyme mining for SNPs in genomes. BMC genomics 2006, 7:30 [http://bio.kuas.edu.tw/snp-rflping/].

II. Chuang LY, Yang CH, Cheng YH, Gu DL, Chang PL, Tsui KH, Chang HW: V-MitoSNP: visualization of human mitochondrial SNPs. BMC bioinformatics 2006, 7:379 [http://bio.kuas.edu.tw/vmitosnp/].

12. Thorisson GA, Smith AV, Krishnan L, Stein LD: The International HapMap Project Web site. Genome research 2005, I 5(1 I I 592-1593 [http://www.hapmap.org].

13. Excoffier L, Slatkin M: Maximum-likelihood estimation of molecular haplotype frequencies in a diploid population. Molecular biology and evolution 1995, I 2(5):921-927.

14. Wigginton JE, Cutler DJ, Abecasis GR: A note on exact tests of Hardy-Weinberg equilibrium. American journal of human genetics 2005, 76(5):887-893.

15. Sherry ST, Ward MH, Kholodov M, Baker J, Phan L, Smigielski EM, Sirotkin K: dbSNP: the NCBI database of genetic variation. Nucleic acids research 200I, 29(1308-31) [http:// www.ncbi.nlm.nih.gov/SNP/].

16. Wheeler DL, Barrett T, Benson DA, Bryant SH, Canese $\mathrm{K}$, Chetvernin V, Church DM, Dicuccio M, Edgar R, Federhen S, et al: Database resources of the National Center for Biotechnology Information. Nucleic acids research 2008:DI3-2I [http:// www.ncbi.nlm.nih.gov].

17. Yang $\mathrm{CH}$, Cheng YH, Chuang LY, Chang HW: SNP-Flankplus: SNP ID-centric retrieval for SNP flanking sequences. Bioinformation 2008, 3(4/47-I 49 [http://bio.kuas.edu.tw/snp-flankplus/].

18. Yang $\mathrm{CH}$, Chuang LY, Cheng YH, Wen $\mathrm{CH}$, Chang PL, Chang HW: SNP ID-info: SNP ID searching and visualization platform. OMICS 2008, I 2(3217-226 [http://bio.kuas.edu.tw/snpid-info].

19. Roberts RJ, Vincze T, Posfai J, Macelis D: REBASE-enzymes and genes for DNA restriction and modification. Nucleic acids research 2007:D269-270 [http://www.rebase.org].

20. Chang HW, Chuang LH, Cheng YH, Hung YC, Wen CH, Gu DL, Yang $\mathrm{CH}$ : Prim-SNPing: a primer designer for cost-effective SNP genotyping. Biotechniques 2009, 46(642I-43I [http:// www.rebase.org].
Publish with Bio Med Central and every scientist can read your work free of charge

"BioMed Central will be the most significant development for disseminating the results of biomedical research in our lifetime. "

Sir Paul Nurse, Cancer Research UK

Your research papers will be:

- available free of charge to the entire biomedical community

- peer reviewed and published immediately upon acceptance

- cited in PubMed and archived on PubMed Central

- yours - you keep the copyright

Submit your manuscript here:

http://www.biomedcentral.com/info/publishing_adv.asp
BioMedcentral 